\title{
Analysis of Excitation and Dead Vibration Modes of Quartz Resonators
}

\author{
Zi-Gui Huang and Zheng-Yu Chen \\ Department of Mechanical Design Engineering, National Formosa University, No. 64, \\ Wenhua Road, Huwei Township, Yunlin County 632, Taiwan \\ Correspondence should be addressed to Zi-Gui Huang; zghuang0119@nfu.edu.tw
}

Received 12 September 2013; Revised 25 December 2013; Accepted 25 December 2013; Published 10 February 2014

Academic Editor: Rama Bhat

Copyright (C) 2014 Z.-G. Huang and Z.-Y. Chen. This is an open access article distributed under the Creative Commons Attribution License, which permits unrestricted use, distribution, and reproduction in any medium, provided the original work is properly cited.

\begin{abstract}
This study uses the finite element method (FEM) to analyze the excitation and dead vibration modes of two-dimensional quartz plates. We first simplify three-dimensional quartz plates with plane strain simplification and then compare the modes of the simplified three-dimensional plates to those of two-dimensional plates. We then analyze quartz vibrating elements of AT-cut plates and SC-cut plates. To understand the regularity of the resonance frequency of plates that are excitable by voltage loading, we compare the natural vibrations of quartz plates with the excitation frequency generated after the plates are excited by voltage loading.
\end{abstract}

\section{Introduction}

Quartz resonators are one of the passive components widely used in the computer, communication, and consumer-electronics industries; they are easily found in computers, electronic instruments, quartz watches, and mobile communication components. Quartz is a type of anisotropic piezoelectric material, and the piezoelectricity was discovered by the brothers Pierre Curie and Jacques Curie in 1880. In 1951, Mindlin expanded plate theory to include crystal plates and the discussion of the frequency of AT-cut quartz plates with consideration of the length-to-thickness ratio [1]. Bechmann then used resonance methods to obtain elastic and piezoelectric constants of different types of man-made quartz [2]. With the improvement in the calculating ability of computers and the use of finite element software, analyses of quartz plates can be found in many recent studies. Wang et al. [3, 4], Yong et al. [5], Patel et al. [6, 7], Patel and Yong [8], and Yang et al. [9] used the finite element method to analyze the piezoelectric vibrations of quartz plate and predict the $Q$ value and equivalent electrical parameters of quartz resonators, in addition to the influence that the mesh impedances of plate grids had on transmitting sound waves.

Since different cutting angles or temperature changes affect the forms of oscillation of quartz, different boundary con-ditions are applied in finite element analysis; the analysis results will then be discussed and compared. Generally, any structure could cause numerous types of natural vibration. One of the oscillation characteristics of quartz plates is the stable resonance frequency generated from the deformation of the quartz material caused by electrodes when applying voltage to the plates. This converse piezoelectric effect converts electrical energy into mechanical energy, and it is different from normal, natural vibrations. In this study, we evaluate the differences between the natural vibrations of quartz plates and the changes in vibration modes resulted from electric field polarization.

\section{Finite Element Analysis on Two-Dimensional Quartz Plates}

2.1. Setting the Size and Boundary Conditions of the Finite Element Model. A finite element analysis simulation software [10] was used in this study to analyze the vibrations of thin quartz plates. The size of the quartz resonators used in this study was $3.495 \mathrm{~mm}$ in length (2a), $0.0638 \mathrm{~mm}$ in thickness $(2 b)$, and $1.805 \mathrm{~mm}$ in width (2c). The AT-cut and SC-cut of anisotropic piezoelectric quartz plates were considered in this paper. We set the boundary condition as the application of 
voltage differences to the upper and lower layers of quartz plates. Figure 1 shows the boundary condition setting of twodimensional models.

2.2. Grid Layout and Frequency Convergence. Thickness shear modes are one of the vibration modes of quartz resonators. The frequencies of thickness shear modes are influenced by the thickness of quartz plates. Therefore, the thickness of grids must be sufficient to make the obtained frequency converge. In general, more grids result in a more precise value. However, to be efficient when analyzing, the number of grids should be just enough to make the frequency convergent. The models in this study come from the analysis results of the number of grids and frequency convergence in a study conducted by Huang and Chen [11].

In their study, Huang and Chen found the appropriate number of grids in terms of grid convergence of the thickness of two-dimensional quartz plate models. When dividing the thickness of a quartz plate $(0.0638 \mathrm{~mm})$ into 10 grids, the error value $(6.9342 \mathrm{ppm})$ they obtained was closer to convergence, compared to when the thickness was divided into 100 grids. Also, when dividing the length of the plate $(3.495 \mathrm{~mm})$ into at least 500 grids, the error value of resonance frequency they obtained dropped to under $30 \mathrm{ppm}$; the value was less compared to that obtained when dividing the length into 1000 grids. Considering the length/grid ratio, the size of each grid was obtained, and the equation was written as 3.495/500 $=6.99 \times 10^{-3}(\mathrm{~mm})$. Based on the grid size, an appropriate number of grids for the width of the plate were calculated to achieve the frequency convergence; the equation was written as $1.805 / 6.99 \times 10^{-3}=258$ (grid). To attain the best frequency convergence result, we divided the length of each model into 500 grids, the width into 260 grids, and the thickness into 10 grids.

2.3. Simplifying Quartz Plate Models. The equations of motion for a piezoelectric plate can be obtained from the variational principle shown in

$$
\int_{t_{0}}^{t} d t \int_{V}\left[\left(T_{i j, i}-\rho \ddot{u}_{j}\right) \delta u_{j}+D_{j, j} \delta \phi\right] d V=0,
$$

where $T_{i j}$ is the stress tensor, $t$ is the time variable, and $\rho$ and $u_{j}$ are the density and displacement vector, respectively. $D_{j}$ is the electric displacement vector and $\phi$ is the electric potential. $V$ is the volume of the piezoelectric plate.

The piezoelectric constitutive relationships among stress, strain, electric field, and electric displacement field in a stresscharge form are expressed as

$$
\begin{gathered}
T_{i j}=c_{i j k l} S_{k l}-e_{k i j} E_{k}, \quad S_{i j}=\frac{1}{2}\left(u_{i, j}+u_{j, i}\right), \\
D_{i}=e_{i j k} S_{j k}+\varepsilon_{i j} E_{j}, \quad E_{i}=-\phi_{, i},
\end{gathered}
$$

where $S_{k l}$ is the strain tensor and $E$ is the electric field. The tensors $c_{i j k l}, e_{k i j}$, and $\varepsilon_{i j}$ are elastic constants, piezoelectric constants (coupling coefficients), and dielectric constants, respectively.

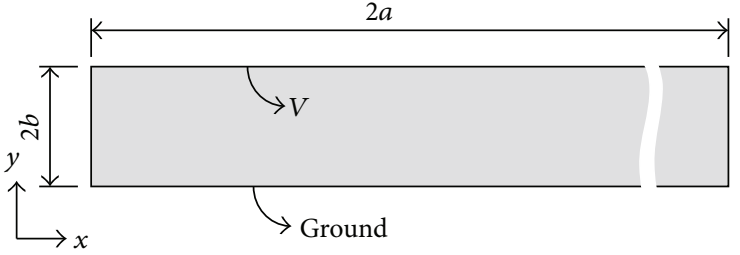

FIGURE 1: Boundary condition setting of two-dimensional quartz plate models.

The stress-strain analysis used to analyze threedimensional models can be divided into two methods for simplification in terms of elasticity: plane stress and plane strain. For anisotropic material like quartz, 2D model based on plane strain assumption does not give accurate results [12]. In this paper, to obtain approximate accurate results for the thickness mode and flexural mode frequencies from the 2D model, the shear strain in the $z$ direction when using the $X Y$ plane strain case and the shear strain in the $x$ direction when using the $Z Y$ plane strain case are not taken into consideration.

The plane stress is mainly used to analyze very thin quartz plates. The external force is evenly distributed through the thickness of the plates, and the stress components do not change with the direction of thickness. Therefore, there are only normal stresses $\sigma_{x}$ and $\sigma_{z}$, and shear stress $\tau_{x z}$ when $X Z$ is considered as the plane in plane stress case. The values of $\sigma_{y}, \tau_{y x}$, and $\tau_{y z}$ would be 0 so that the plane stress case cannot be used to discuss the thickness shear vibration modes.

The plane strain is mainly applied to analyze longer pillars. The cross section of a pillar and the longitudinal force do not change with length. When the $z$-axis is the major axis, then the displacement along the $z$-axis would always be 0 . Thus, there are only normal strains $\varepsilon_{x}$ and $\varepsilon_{y}$ and shear strain $\gamma_{x y}$ when $X Y$ is considered as the plane in the plane strain case. Here, strains $\varepsilon_{z}, \gamma_{y z}$, and $\gamma_{x z}$ would not exist.

Thickness shear modes are the main source of resonation in quartz resonators for specific cuts of quartz; they can be seen as horizontal shearing motions along the $x$ or $z$ direction when the normal of plane is $y$. Though thin plate resonators tend to apply the simplification method of plane stress, the stress and shear stress of the thickness direction are not included in a plane stress analysis. This does not meet the requirements of two-dimensional quartz plate analysis. Therefore, we used plane strain analysis to analyze the two-dimensional quartz plate and discuss the resonance characteristics of the plate's $X Y$ plane. To simplify the plate, or to ignore the influence that $z$-axis had on the plane, we made the length of the $z$-axis much longer than those of the $x$ and $y$-axes. The $X Y$ plane of the simplified three-dimensional resonator is shown as Figure 2.

2.4. Comparison between Three-Dimensional and TwoDimensional Quartz Plate Modes. The simplification of three-dimensional models helps to improve the analysis efficiency of the finite element method. However, according to the grid convergence information in the study conducted by Huang and Chen, the size of each grid of the model 
TABLE 1: Sizes and grid layouts of the three- and two-dimensional models.

\begin{tabular}{lccr}
\hline SC-cut & Model & $\begin{array}{c}\text { Dimension } \\
(L \times W \times T)(\mathrm{mm})\end{array}$ & $\begin{array}{c}\text { Mesh numbers } \\
(L \times W \times T)\end{array}$ \\
\hline \multirow{2}{*}{ Case 1} & 2D $(X Y$ plane $)$ & $3.53 \times 0.0638(L \times T)$ & $500 \times 10(L \times T)$ \\
& 3D & $3.53 \times 1.805 \times 0.0638$ & $500 \times 1 \times 10$ \\
\hline \multirow{2}{*}{ Case 2 } & 2D (ZY plane $)$ & $1.85 \times 0.0638(W \times T)$ & $260 \times 10(W \times T)$ \\
& 3D & $3.495 \times 1.85 \times 0.0638$ & $1 \times 260 \times 10$ \\
\hline
\end{tabular}

TABLE 2: Comparison of the error values of three- and twodimensional models' mode frequency.

\begin{tabular}{llcccc}
\hline SC-cut & \multicolumn{2}{c}{$3 \mathrm{D}(\mathrm{MHz})$} & \multicolumn{2}{c}{$2 \mathrm{D}(\mathrm{MHz})$} & Error $(\%)$ \\
\hline \multirow{4}{*}{ Case 1} & $p_{1}$ & 28.0525 & $m_{1}$ & 28.0725 & 0.069513 \\
& $p_{2}$ & 28.145 & $m_{2}$ & 28.215 & 0.248712 \\
& $p_{3}$ & 28.25 & $m_{3}$ & 28.33 & 0.283186 \\
\hline \multirow{3}{*}{ Case 2 } & $a_{1}$ & 30.65 & $b_{1}$ & 30.67 & 0.065253 \\
& $a_{2}$ & 31.005 & $b_{2}$ & 30.938 & -0.21609 \\
& $a_{3}$ & 31.39 & $b_{3}$ & 31.435 & 0.143358 \\
\hline
\end{tabular}

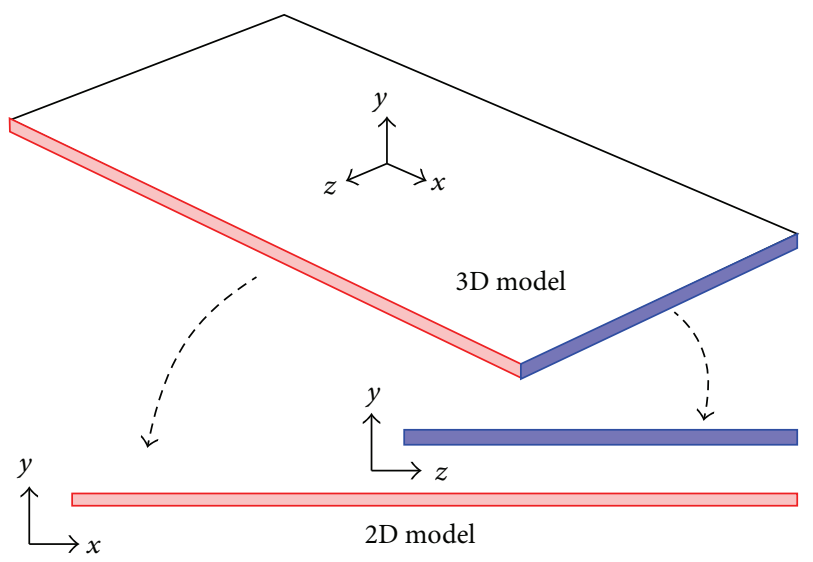

FIGURE 2: Simplification of quartz plate model.

dimensions must be smaller than $7 \times 10^{-3} \mathrm{~mm}$ to achieve frequency convergence [11]. This could be very difficult to realize when analyzing a three-dimensional model. Considering the directional effects of the length, the width, and the thickness of a three-dimensional model produces extremely complicated oscillating curves.

To compare a three-dimensional model with the $X Y$ plane of a two-dimensional model, we set the grid layout of the three-dimensional model to 500 grids for the length, 1 grid for the width, and 10 grids for the thickness. We set the grid layout of the other three-dimensional model to 1 grid for the length, 260 grids for the width, and 10 grids for the thickness when comparing it with the $Z Y$ plane of the two-dimensional model. This type of grid layout reduced the complexity of the three-dimensional models and facilitated the comparison of the frequency curves of the three-dimensional models to those of the two-dimensional models. The sizes and grid layouts of the models in this study are shown in Table 1.

Figures 3 and 4, respectively, show the frequency responses obtained from analyzing the models in Case 1 and Case 2. We selected three different modes from the curves to analyze; they were flexural modes (indicated by 1 ), thickness shear modes (indicated by 2), and coupled resonant modes (indicated by 3 ). From the mode shapes corresponding to the noticeable peak values in Figure $3\left(m_{1}, m_{2}, m_{3}, p_{1}, p_{2}\right.$, and $p_{3}$ ), we found that we could still obtain similar modes from the two-dimensional model. The error values of Case 1 are shown in Table 2. Though the number of grids was set to 1 for the width of the three-dimensional model, there were still some oscillations caused by the width effect. In other words, the advantage of using a two-dimensional model for analysis is that the influence of the width direction of the plate can be eliminated. The oscillation modes of the $X Y$ plane can then be specifically discussed.

The same result was discovered when using the models in Case 2 to analyze the $Z Y$ plane; we obtained the result by comparing the mode shapes corresponding to the noticeable peak values in Figure $4\left(a_{1}, a_{2}, a_{3}, b_{1}, b_{2}\right.$, and $\left.b_{3}\right)$. The difference was that the baseband of the $Z Y$ direction was located at a higher frequency $(31.005 \mathrm{MHz})$ and the baseband of the $X Y$ direction was located at a lower frequency $(28.145 \mathrm{MHz})$. Thus, the resonant mode of the $Z Y$ direction was easily affected by the coupled resonant mode of the $X Y$ direction; this caused the resonant mode of the $Z Y$ direction to separate and produce incomplete oscillations. This is shown as Mode $a_{3}$ in Figure 4.

In this section, we used SC-cut three-dimensional models and two-dimensional models to compare the mode oscillations. When using three-dimensional models for analysis, we found it difficult to examine the oscillation characteristics of the plates due to the complicated oscillating modes resulting from the natural vibrations of the three directions. Therefore, we set the number of grids to 1 for each single direction (either the length or the width) to decrease the degree of interference. Through analysis of the two-dimensional models, we found that the mode shapes after simplification corresponded to those of the three-dimensional models. The average error value fell within the range of \pm 0.02 to $0.3 \%$. In other words, we could replace the three-dimensional vibration analysis with a two-dimensional plane strain analysis when only examining the oscillation characteristics of the thickness direction. This not only effectively reduced the interference from the third direction but also significantly improved the efficiency of analysis. Thus, we used the two-dimensional models for the following analyses.

\section{Excitation and Dead Vibration Modes of Quartz Resonators}

Through the analysis of finite element software, we found that quartz plates produced numerous types of oscillation modes 


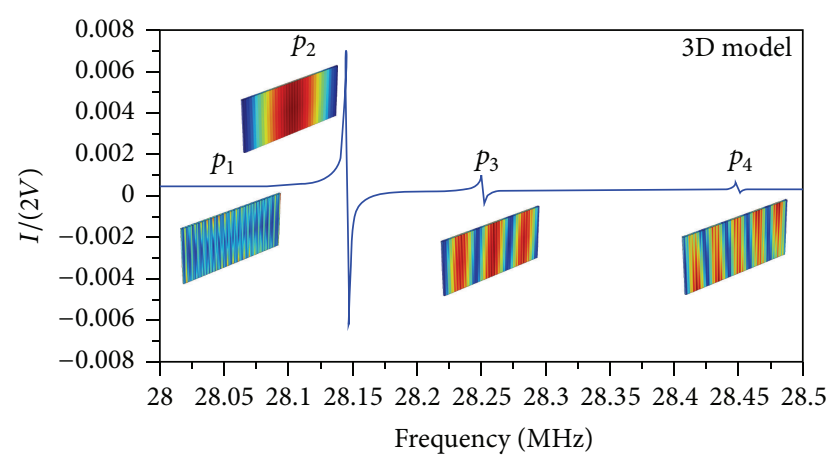

(a)

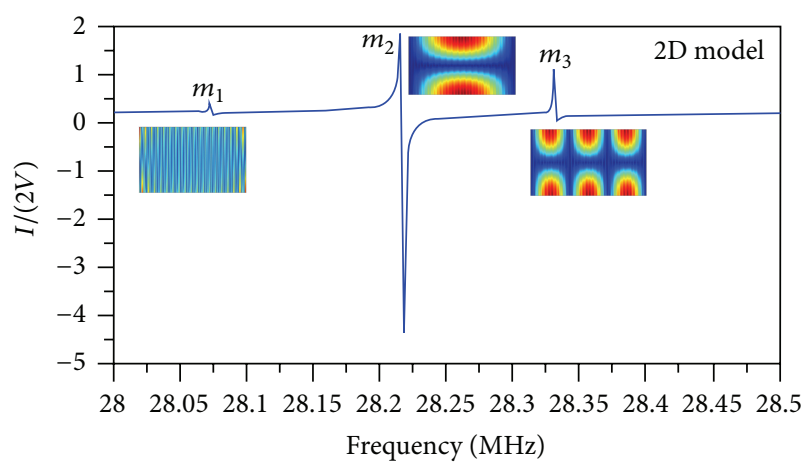

(b)

Figure 3: Comparison of the frequency response of the SC-cut three-dimensional plate and the SC-cut two-dimensional plate $(X Y$ plane).

when excited by an electric field. The patterns of these oscillation modes were exactly the same as those generated from natural oscillations. But the resonances at certain frequencies became minimal after being excited by the voltage loading. Thus, we considered the resonances which had a frequency width of less than $2500 \mathrm{~Hz}$ as failure resonant frequencies. We further discussed whether the mode characteristics of AT-cut and SC-cut plates could be excited by the electric field and the regularity of the frequencies.

3.1. Analysis Results and Discussion (AT-Cut). The analysis of quartz plates can be divided into two parts; one is the analysis of natural vibration frequencies, and the other is the analysis of excited vibration frequencies caused by voltage loading. When the sizes of quartz resonators differ, their resonance modes change in different ways. Figure 5 shows the dispersion curves of the resonance modes when AT-cut plates of different sizes vibrate naturally and when they are excited by electrodes.

In Figure 5, the horizontal axis represents the length-tothickness ratio $(a / b)$, and the longitudinal axis represents the frequency value. The range of frequency is between $25 \mathrm{MHz}$ and $27 \mathrm{MHz}$. The curves composed of solid dots denote the natural resonant modes of quartz plates, and the curves composed of empty dots denote the resonant modes of plates excited by voltage loading. By slightly modifying the size of the plates, it is easy to find differences between the plates that were excited by the resonant nodes and those plates that were

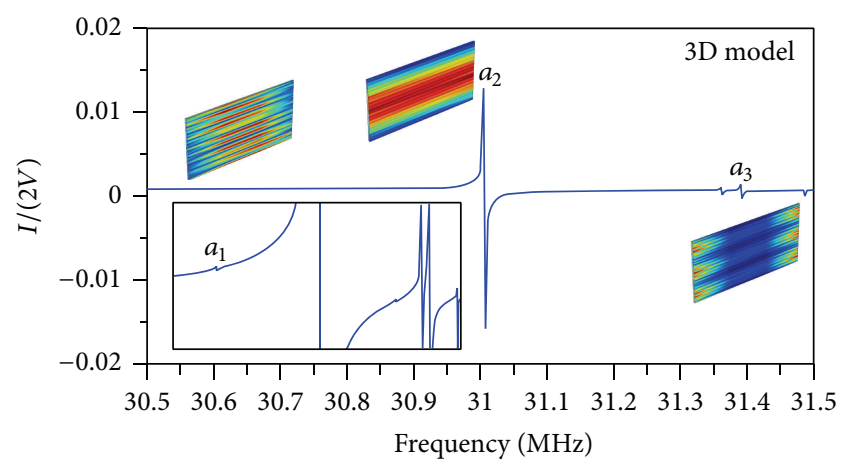

(a)

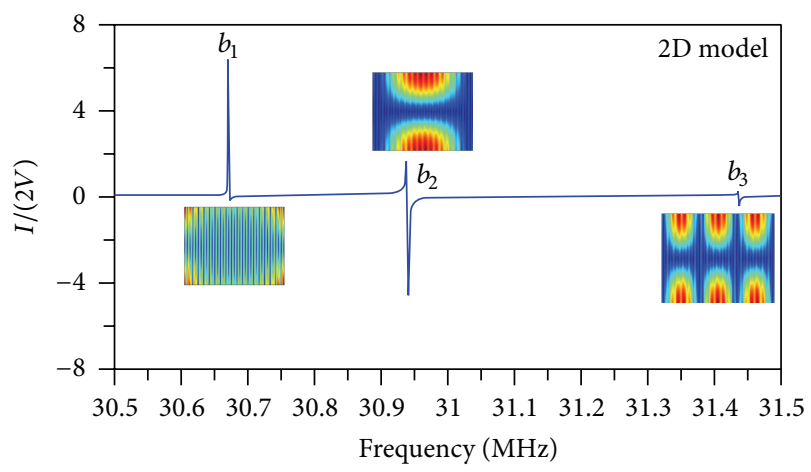

(b)

FIGURE 4: Comparison of the frequency response of the SC-cut three-dimensional plate and the SC-cut two-dimensional plate $(Z Y$ plane).

not. The longitudinal line in Figure 5 represents the location of the original size of the quartz resonator, and the original size was further analyzed. Figure 6 shows the comparison of the natural vibrations and the excited vibrations of the original plate size.

The goal is to compute the susceptance (the imaginary part of the admittance) for a frequency range around the eigenfrequencies of the structure. In Figure 6, the horizontal axis represents the frequency, and the longitudinal axis represents the susceptance. The line denotes the frequency response curve generated from the vibrations after the plate is excited by the voltage loading, and the solid dots are the resonance frequencies of the plate's natural vibrations. According to Figure 6, the plate produces 16 natural resonant modes when the frequency ranges between $25 \mathrm{MHz}$ and $27 \mathrm{MHz}$. However, there are only 7 modes of excited vibrations generated in this range when exciting the plate with voltage loading. Therefore, when applying voltage to the piezoelectric plate, some modes generate resonance frequencies of low impedance when being excited by voltage loading. Yet, some natural frequencies of the plate are unexcitable by voltage loading and subsequently cause resonant vibration failure. In brief, only some resonant modes can be excited among the plate resonances generated from the converse piezoelectric effect.

To determine the regularity among the resonant modes generated from both the plate's natural vibrations and the excited vibrations, we had a discussion on the resonant modes 


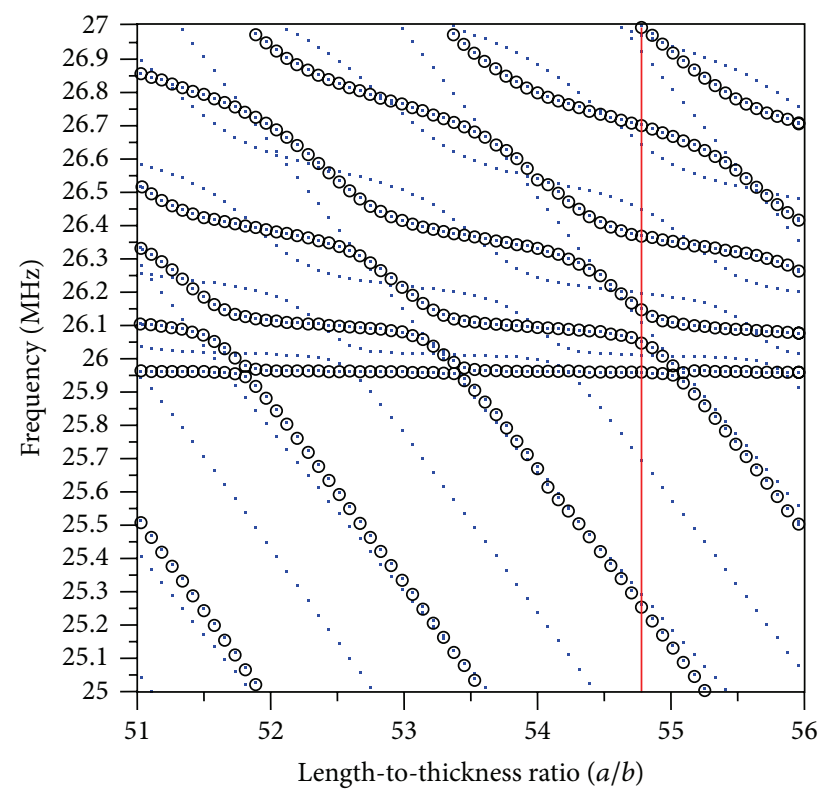

FIGURE 5: Dispersion curves of the resonance modes of different sized plates vibrating naturally and being excited by electrodes (ATcut, $X Y$-plane).

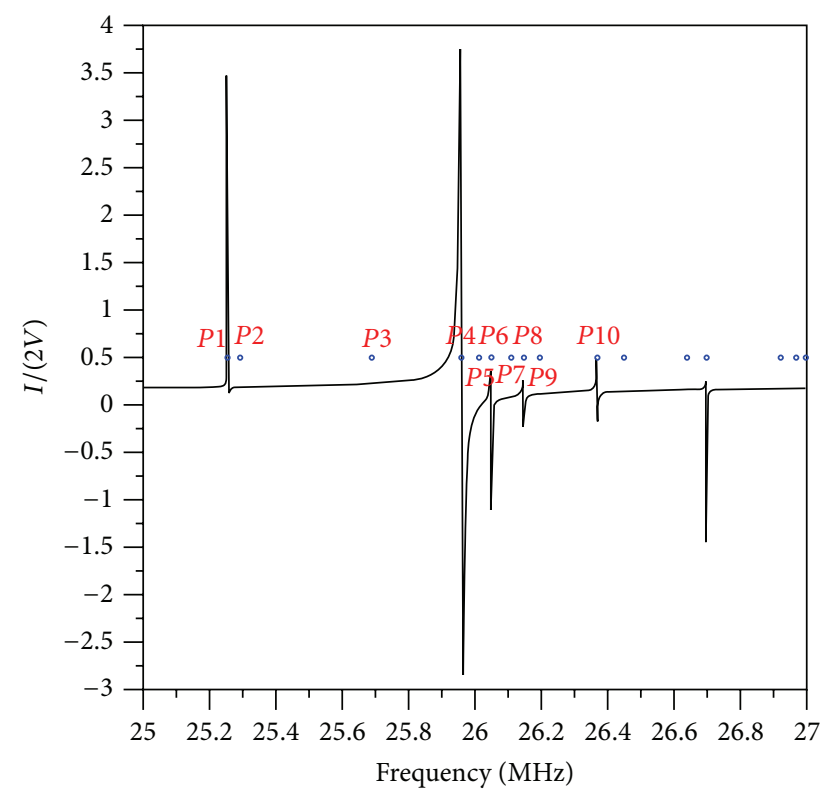

FIgURE 6: Comparison of the plate's natural and excited vibrations.

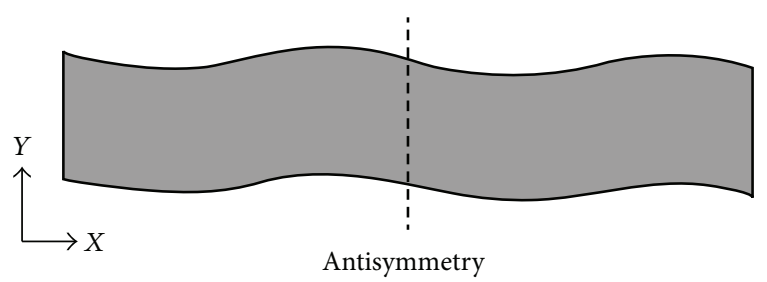

Figure 7: Antisymmetric resonant mode.

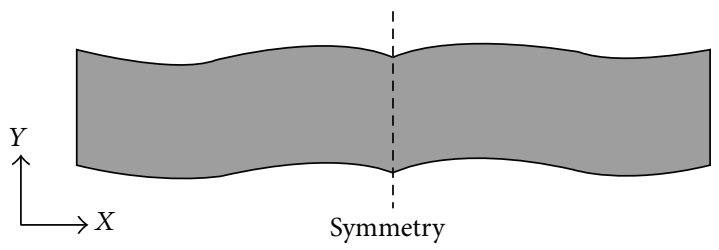

FIGURE 8: Symmetric resonant mode.

in Figure 6. By observing the 15 modes within the frequency range, we found several characteristics of vibrations which could be excited by voltage loading.

Among the vibration modes of two-dimensional quartz plates, there are four basic modes. They are the flexural mode, the thickness shear mode, the coupled mode of the flexural and thickness shear modes, and the extension mode. These four modes can be further divided into symmetric and antisymmetric resonant modes in terms of their shape (as shown in Figures 7 and 8). After categorizing the resonance characteristics of the modes, preparations are complete for determining whether the resonant modes can be excited.

First, we evaluated the symmetry of each mode by looking at the mode shapes in Figure 6. The mode shapes of $P 1$ to $P 10$ in Figure 6 were then taken out to develop Figure 9. Among the ten modes, $P 3, P 5, P 7$, and $P 9$ were symmetric resonant modes; $P 1, P 4, P 6, P 8$, and $P 10$ were categorized as antisymmetric resonant modes. By evaluating the resonant modes of different symmetric characteristics, we found some consistent phenomena. When the mode shapes were considered antisymmetric, they could be excited; however the symmetric resonant modes could not be excited. $P 2$ and $P 7$ appeared neither symmetric nor antisymmetric, but according to the analysis, neither could be excited. This was further examined in the following portion of the study.

3.2. Analysis Results and Discussion (SC-Cut). The vibrations of piezoelectric plates represent the coupling effects of the force field and the electric field. Therefore, the material elasticity matrix of the force field, the dielectric constant matrix of the electric field, and the piezoelectric constant matrix of the electromechanical coupling must be provided in the analysis. Through the simplification of quartz plates, we ran the plane strain analysis on the $X Y$ plane and the $Z Y$ plane of two-dimensional models. However, when running the analysis on the $Z Y$ plane of AT-cut quartz plates, the piezoelectric constants of the $Z Y$ direction, $e_{222}, e_{233}, e_{223}$, $e_{322}, e_{333}$, and $e_{323}$, were always 0 ; we could not analyze the piezoelectric plate oscillations of the $Z Y$ plane. Thus, we additionally analyzed the resonant modes of the $X Y$ and $Z Y$ planes of SC-cut plates. We then compared the locations of the two thickness shear modes and the regularities of their excited vibration frequencies and frequencies of failure.

The material parameters of SC-cut plates stand for the application of a different kind of quartz plates, and these plates have better adaptability to temperature changes. When the temperature changes, the error value of the thicknessshear frequency is lower compared to that of AT-cut plates. The size of the SC-cut quartz plates used in this study 
$P 1$ : flexural mode-antisymmetric resonance

eigfreq_smpp $n(19)=2.525505 e 7$ surface: total displacement $(\mathrm{m})$

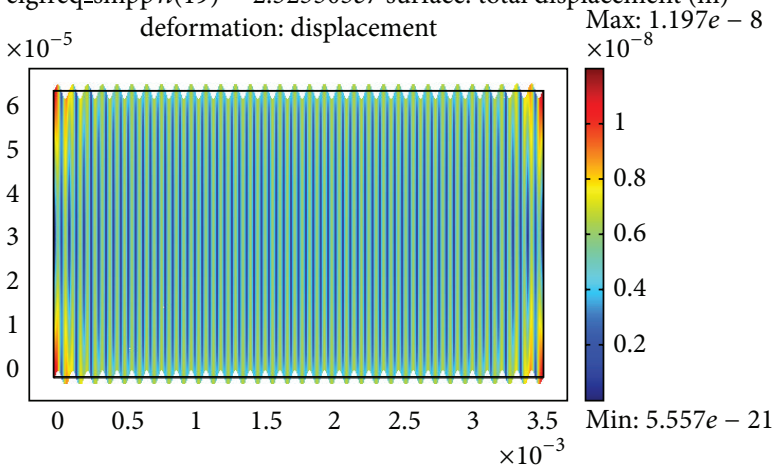

(a)

P3: flexural mode-symmetric resonance eigfreq_smpp $n(21)=2.568837 e 7$ surface: total displacement $(\mathrm{m})$

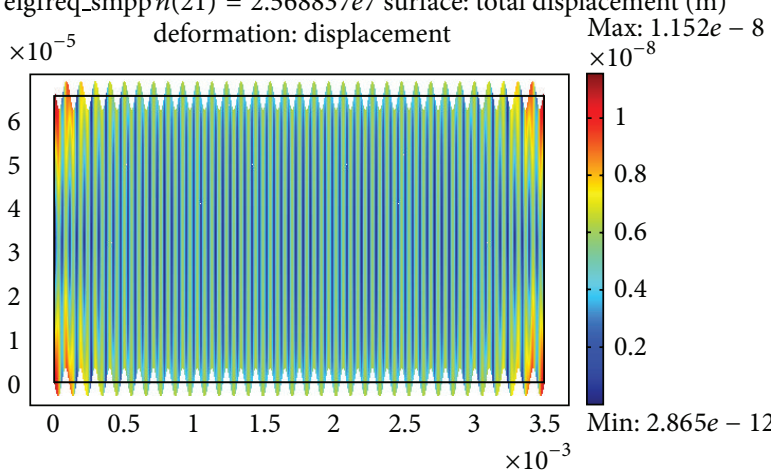

(c)

P5: coupled mode-symmetric resonance

eigfreq_smpp $n(23)=2.601065 e 7$ surface: total displacement $(\mathrm{m})$

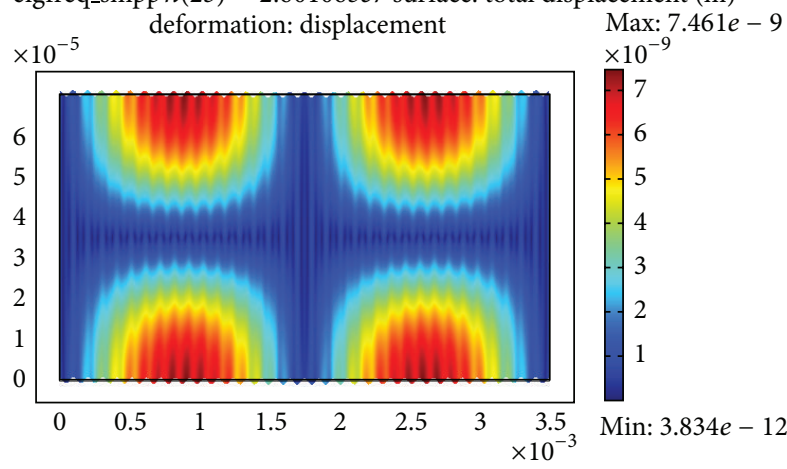

(e)

P7: extension mode-symmetric resonance eigfreq $\operatorname{smpp} n(25)=2.610793 e 7$ surface: total displacement $(\mathrm{m})$

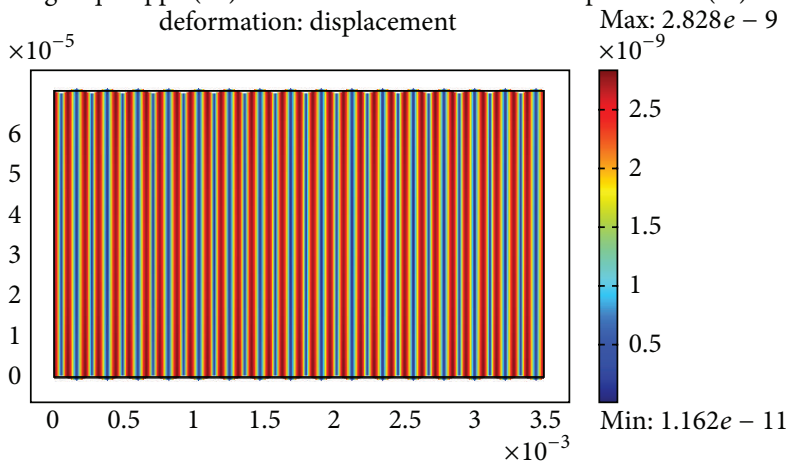

(g)
$P 2$ : extension mode-symmetric resonance

eigfreq_smpp $n(20)=2.529264 e 7$ surface: total displacement $(\mathrm{m})$

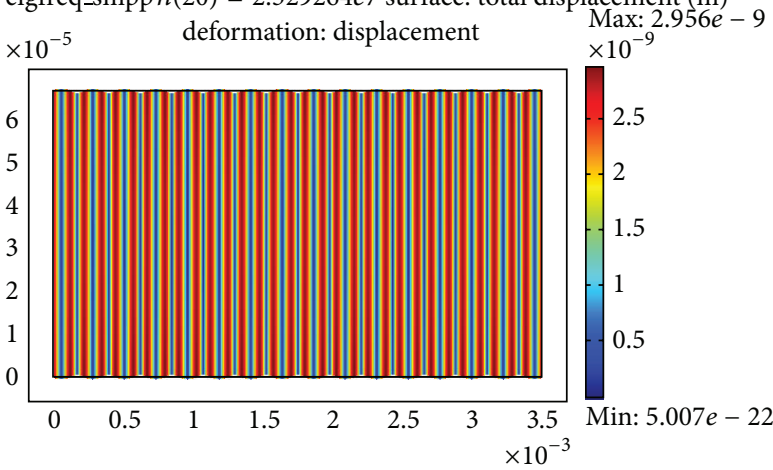

(b)

P4: thickness shear mode-antisymmetric

eigfreq_smpp $n(22)=2.595844 e 7$ surface: total displacement $(\mathrm{m})$

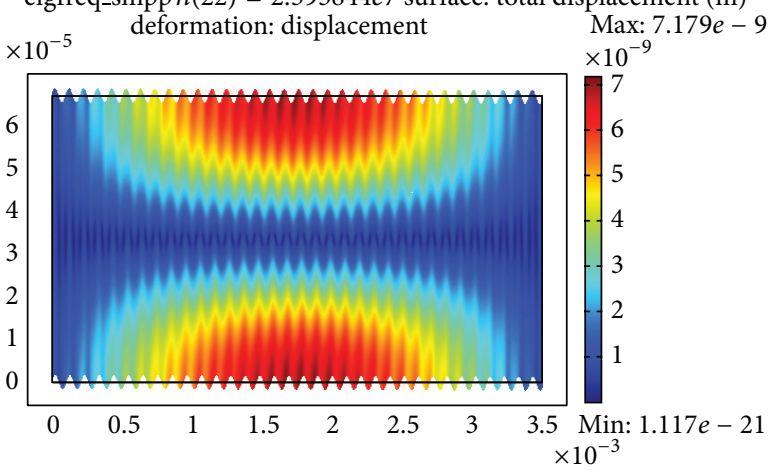

(d)

P6: coupled mode-antisymmetric resonance eigfreq_smpp $n(24)=2.604946 e 7$ surface: total displacement $(\mathrm{m})$

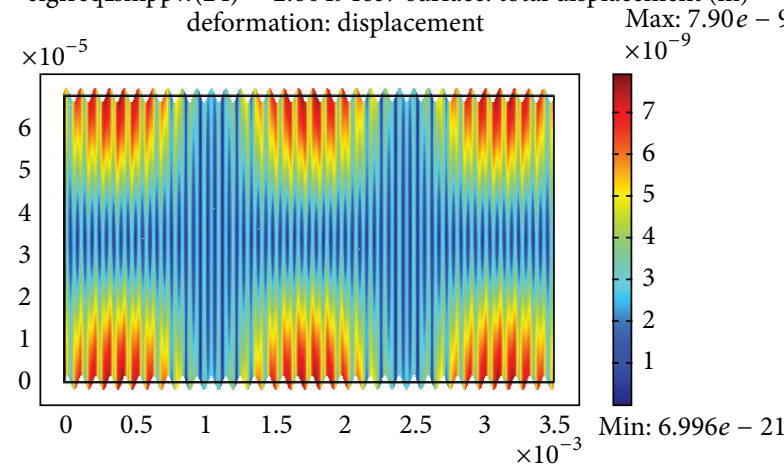

(f)

P8: coupled mode-antisymmetric resonance eigfreq_smpp $n(26)=2.61467 e 7$ surface: total displacement $(\mathrm{m})$

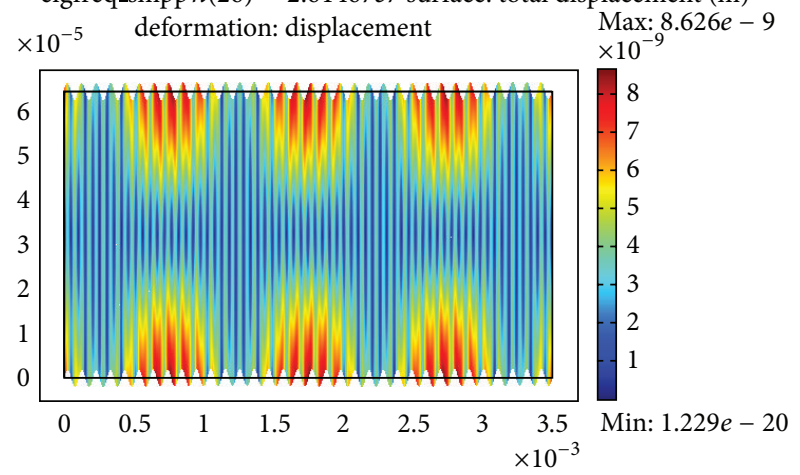

(h)

Figure 9: Continued. 


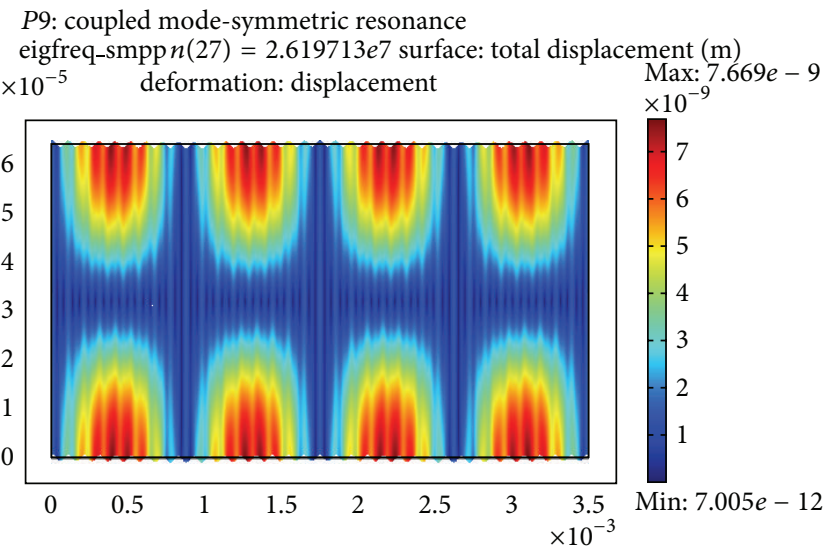

(i)

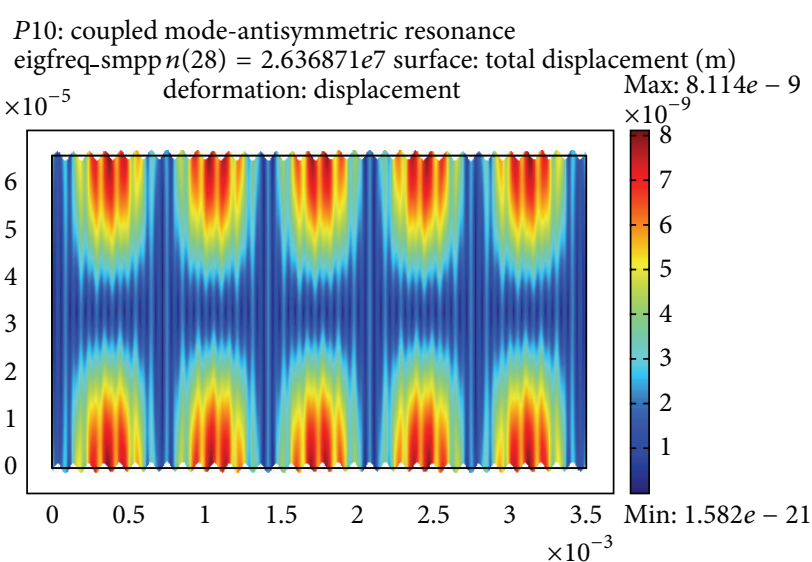

(j)

Figure 9: Mode shapes ( $P 1$ to $P 10)$.

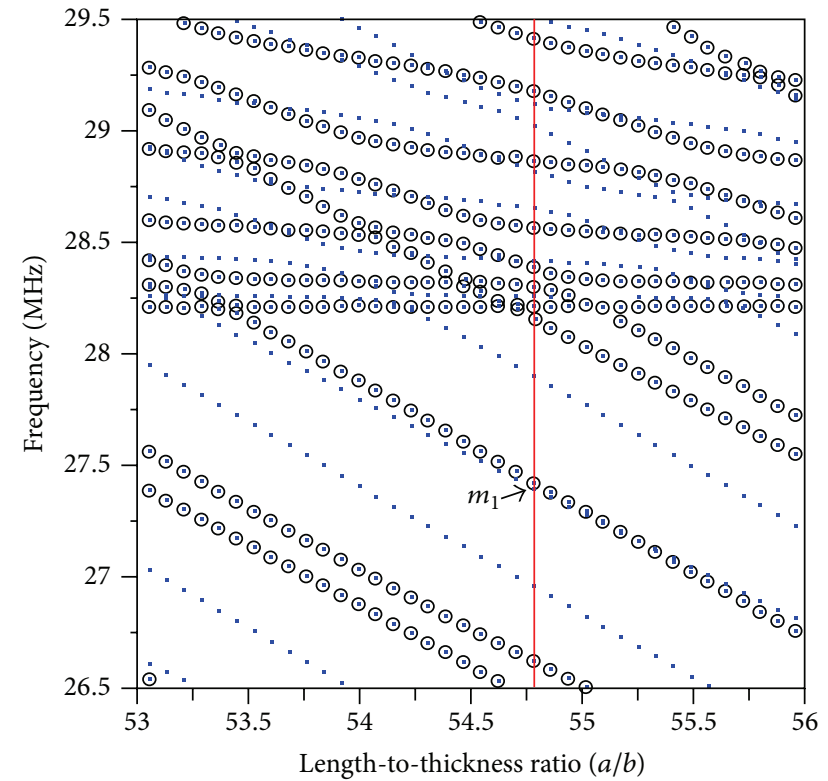

FIGURE 10: Dispersion curves of the resonant modes of different sized plates vibrating naturally and being excited by electrodes (SCcut, $X Y$-plane).

is $3.53 \mathrm{~mm}$ in length $(2 a), 0.0638 \mathrm{~mm}$ in thickness $(2 b)$, and $1.85 \mathrm{~mm}$ in width $(2 c)$. According to the analyses, the thickness shear mode of $X Y$ plane was $28.21586 \mathrm{MHz}$ and that of $Z Y$ plane was $30.93937 \mathrm{MHz}$. We set frequency ranges of $3 \mathrm{MHz}$ above and below the two basebands for the $X Y$ and $Z Y$ planes to develop frequency curves of different sized plates (as shown in Figures 10 and 11).

The purpose of analyzing the vibrations of SC-cut plates was to compensate for the incapability of analyzing the $Z Y$ plane of AT-cut plates. Besides, we used the analysis results of SC-cut plates to decide whether the method used to determine the excited vibration frequencies and dead vibration frequencies in previous section was appropriate. We found that when the resonant modes were antisymmetric, they could also be excited and the modes that were considered

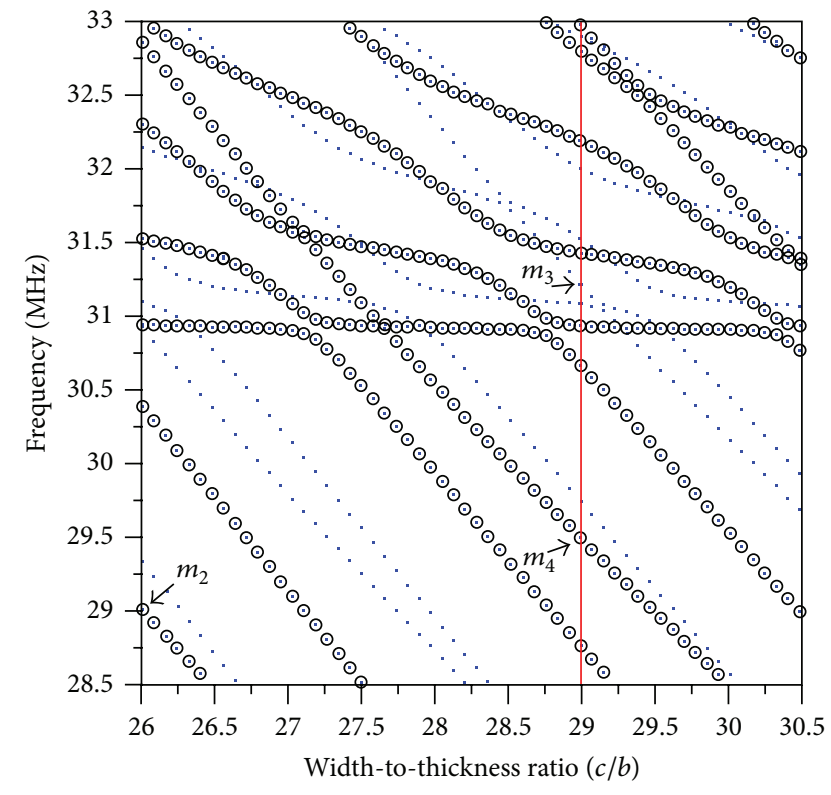

FIGURE 11: Dispersion curves of the resonant modes of different sized plates vibrating naturally and being excited by electrodes (SC-cut, $Z Y$-plane).

symmetric could not be. This proved the suitability of the method used previously. However, something different occurred when analyzing only the $X Y$ plane of AT-cut plates. In this case, we could not be certain about the requirements for exciting the extensional vibration modes, since the five curves of extensional vibration modes in Figure 5 were neither symmetric nor antisymmetric. Moreover, none of them could be excited. Therefore, we needed more data to find out the requirements for exciting extensional vibration modes.

We selected 4 extensional vibration mode shapes $\left(m_{1}\right.$ to $m_{4}$ ) from the analyses of the $X Y$ and $Z Y$ planes of SCcut plates to develop Figure 12. The mode shapes of $m_{1}$ and $m_{3}$ were symmetric, and the mode shapes of $m_{2}$ and $m_{4}$ were antisymmetric. After analysis, the results of exciting 


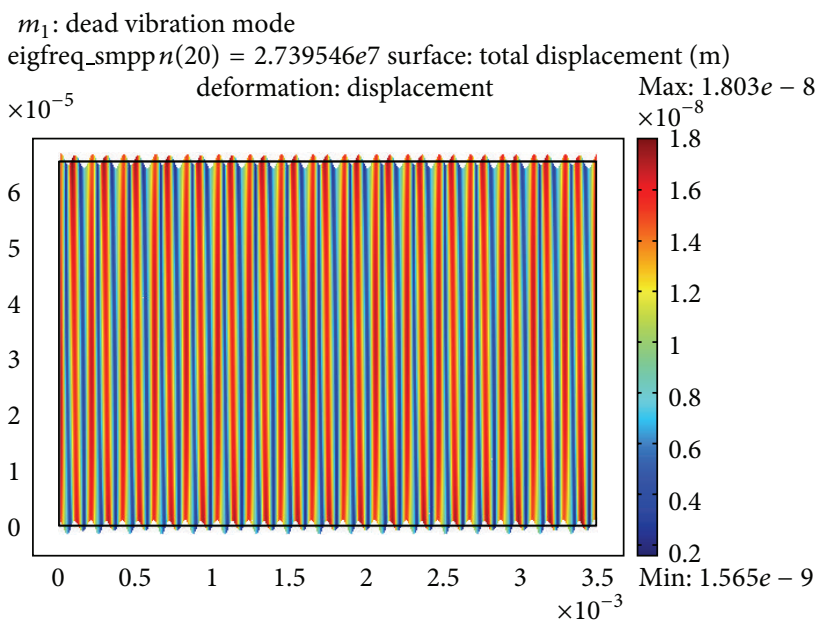

(a)

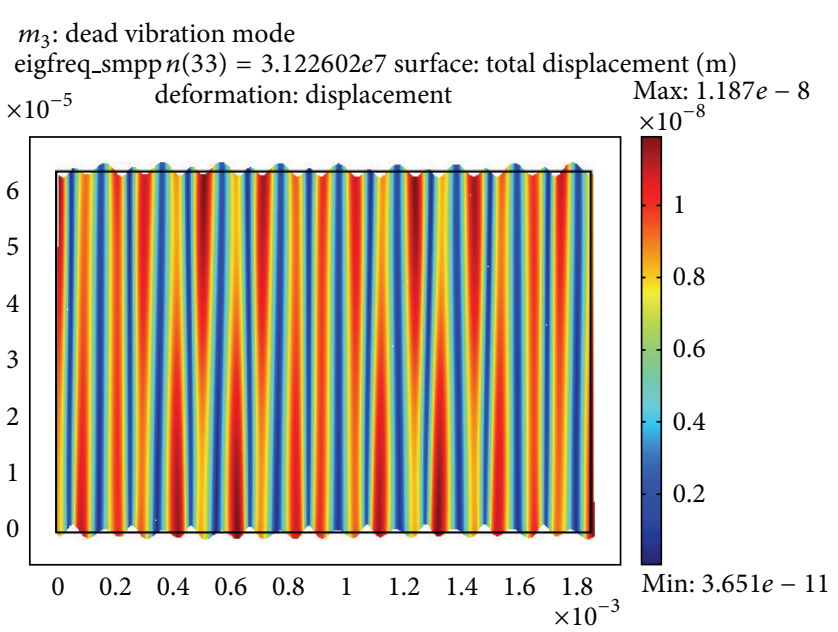

(c)

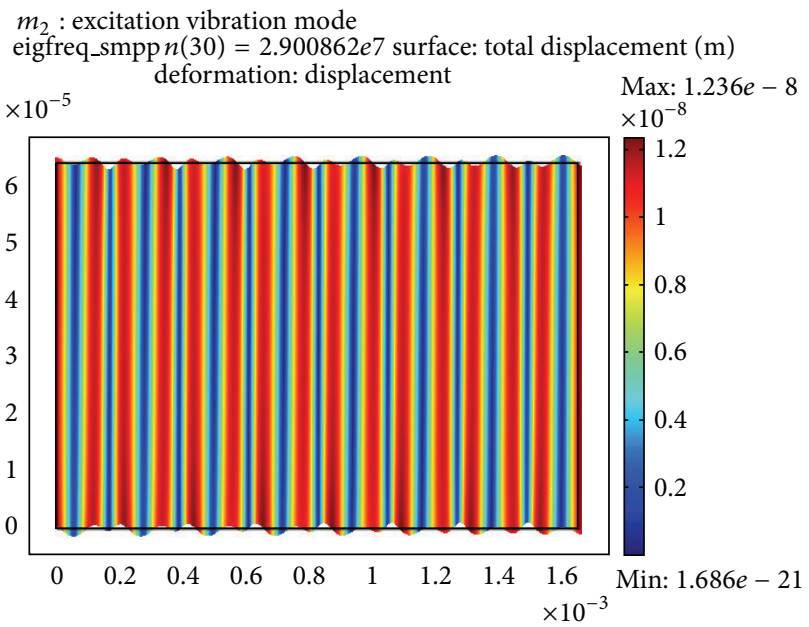

(b)

$m_{4}$ : excitation vibration mode eigfreq_smpp $n(28)=2.949939 e 7$ surface: total displacement $(\mathrm{m})$

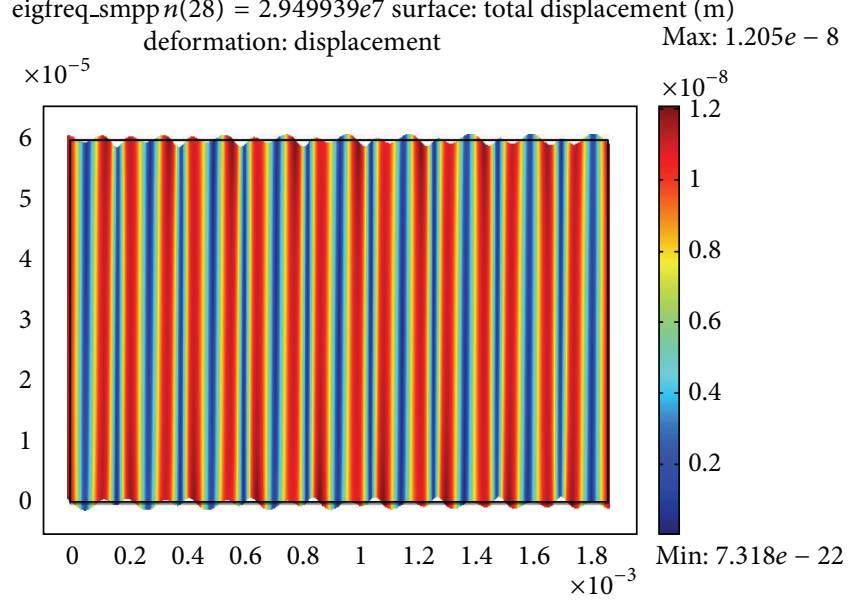

(d)

FIGURE 12: Extensional vibration modes (in SC-cut).

these modes with voltage corresponded to those mentioned in Section 3.1. Thus, we believe that the requirements for exciting extensional vibration modes are the same as those mentioned in Section 3.1, where the mode shapes can be excited when they are antisymmetric.

\section{Conclusion}

In this study, we discuss the resonances of quartz plates. We first simplify our models with plane strain analysis and then analyze the resonances of $X Y$ and $Z Y$ planes of our two-dimensional models. Quartz resonators are considered as frequency components; therefore, the number of grids becomes especially important when conducting the finite element analyses. Based on an earlier study [10], we set the grid layout of the models as 500 grids for the length, 260 grids for the width, and 10 grids for the thickness to achieve the best frequency convergence. We additionally analyze the $X Y$ and
$Z Y$ planes of SC-cut material parameters, since the $Z Y$ plane of AT-cut plates cannot be simplified.

The continuous stable oscillations of quartz resonators come from the converse piezoelectric effects when applying voltage to piezoelectric plates. However, when analyzing the oscillations of quartz plates, we notice that the excited quartz plates can only produce certain resonant modes; other resonant modes are considered as having failed, since they are not excitable by voltage loading. We use the finite element software to analyze the free oscillations and excited vibrations of quartz plates. By comparing the characteristics of resonant modes, we conclude that the resonant modes of quartz plates can be excited when the mode shapes are antisymmetric; when the mode shapes of the plates are symmetric, they cannot be excited. The difference in the surface charge density for symmetric modes obtained from free vibration approximated zero and hence cannot be excited by the voltage loading under the electrode configuration studied in this 
paper. This is in accordance with the experimental study of resonators and of the general result of Lewis' paper [13].

\section{Conflict of Interests}

The authors declare that there is no conflict of interests regarding the publication of this paper.

\section{Acknowledgment}

The authors thank the National Science Council (Project nos. NSC 100-2221-E-150-030 and NSC 101-2628-E-150-001-MY3) for funding. The support of the council has allowed this study to be conducted.

\section{References}

[1] R. D. Mindlin, "Thickness-shear and flexural vibrations of crystal plates," Journal of Applied Physics, vol. 22, no. 3, p. 316, 1951.

[2] R. Bechmann, "Elastic and piezoelectric constants of alpha-quartz," Physical Review, vol. 110, no. 5, pp. 1060-1061, 1958.

[3] J. Wang, Y. K. Yong, and T. Imai, "Finite element analysis of the piezoelectric vibrations of quartz plate resonators with higherorder plate theory," International Journal of Solids and Structures, vol. 36, no. 15, pp. 2303-2319, 1999.

[4] J. Wang, Y.-K. Yong, and T. Imai, "On the accuracy of mindlin plate predictions for the frequency-temperature behavior of resonant modes in AT- And SC-Cut quartz plates," IEEE Transactions on Ultrasonics, Ferroelectrics, and Frequency Control, vol. 46, no. 1, pp. 1-13, 1999.

[5] Y.-K. Yong, M. Patel, J. Vig, and A. Ballato, "Effects of electromagnetic radiation on the Q of quartz resonators," IEEE Transactions on Ultrasonics, Ferroelectrics, and Frequency Control, vol. 56, no. 2, pp. 353-360, 2009.

[6] M. S. Patel, Y. K. Yong, and M. Tanaka, "Drive level dependency in quartz resonators," International Journal of Solids and Structures, vol. 46, pp. 1856-1871, 2009.

[7] M. S. Patel, Y.-K. Yong, and M. Tanaka, "Theory and experimental verifications of the resonator $\mathrm{Q}$ and equivalent electrical parameters due to viscoelastic and mounting supports losses," IEEE Transactions on Ultrasonics, Ferroelectrics, and Frequency Control, vol. 57, no. 8, pp. 1831-1839, 2010.

[8] M. S. Patel and Y.-K. Yong, "Conceptual design of a high-q, 3.4-GHz thin film quartz resonator," IEEE Transactions on Ultrasonics, Ferroelectrics, and Frequency Control, vol. 56, no. 5, pp. 912-920, 2009.

[9] Z. Yang, Y. Hu, J. Wang, and J. Yang, "Nonlinear coupling between thickness- shear and thickness-stretch modes in a rotated y-cut quartz resonator," IEEE Transactions on Ultrasonics, Ferroelectrics, and Frequency Control, vol. 56, no. 1, pp. 220-224, 2009.

[10] COMSOL Multiphysics, Structural Mechanics, Manual, Comsol AB, Stockholm, Sweden, 2013.

[11] Z.-G. Huang and Z.-Y. Chen, "Design analysis of miniature quartz resonator using two-dimensional finite element model," IEEE Transactions on Ultrasonics, Ferroelectrics, and Frequency Control, vol. 58, no. 6, pp. 1145-1154, 2011.

[12] P. C. Y. Lee and R. Huang, "Mechanical effects of electrodes on the vibrations of quartz crystal plates," IEEE Transactions on Ultrasonics, Ferroelectrics, and Frequency Control, vol. 49, no. 5, pp. 612-625, 2002.
[13] J. A. Lewis, "The effect of driving electrode shape on the electrical properties of piezoelectric crystals," Bell System Technical Journal, vol. 40, no. 5, pp. 1259-1280, 1961. 

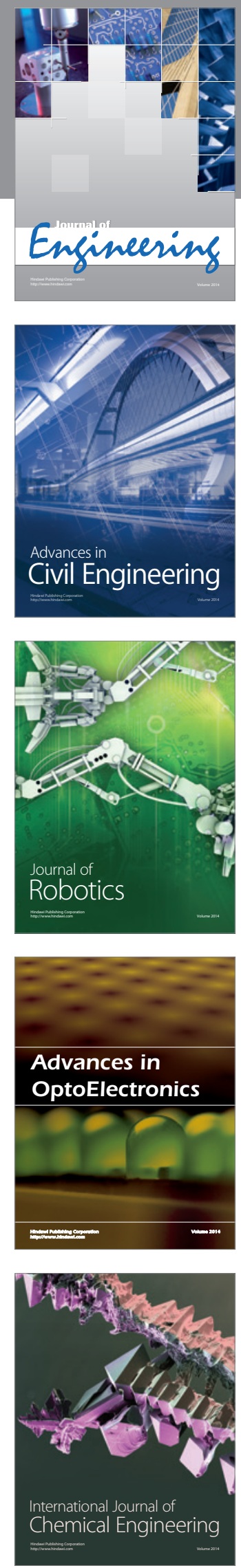

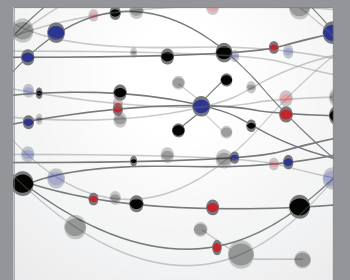

The Scientific World Journal
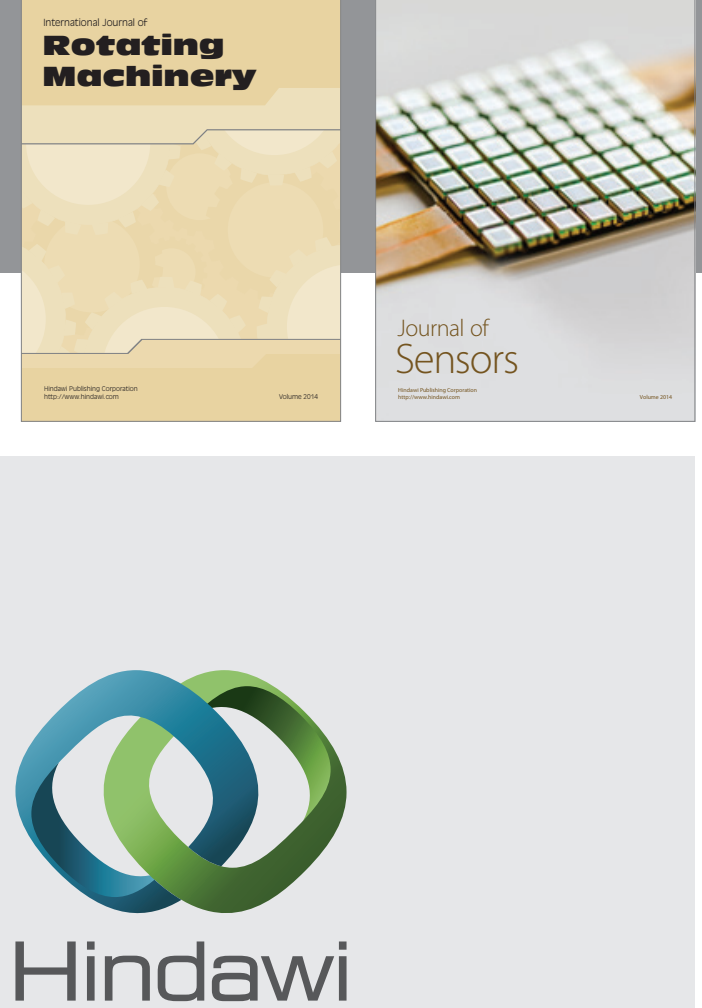

Submit your manuscripts at http://www.hindawi.com
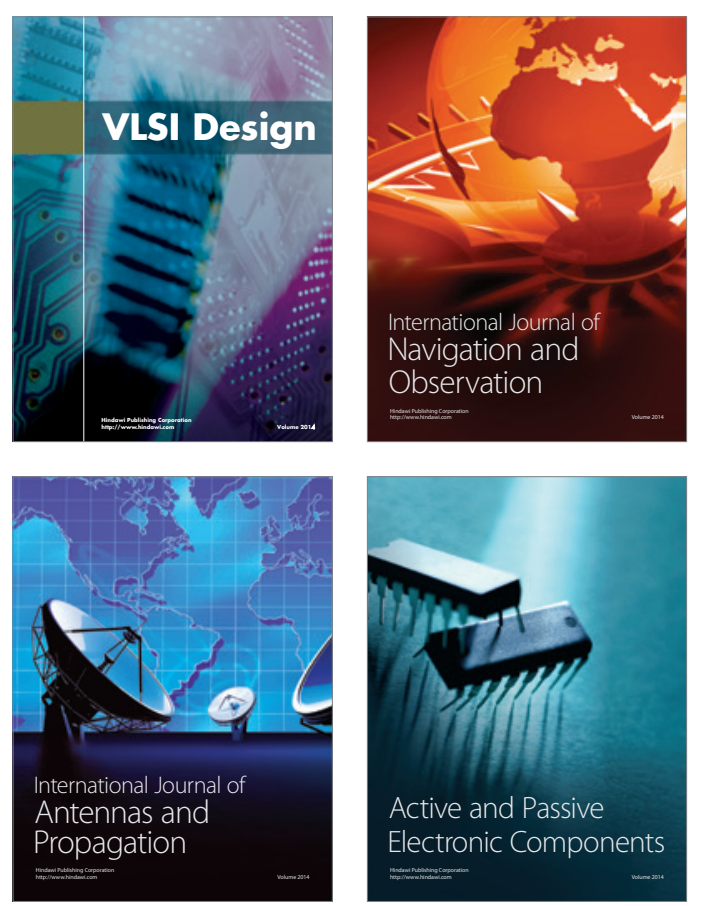
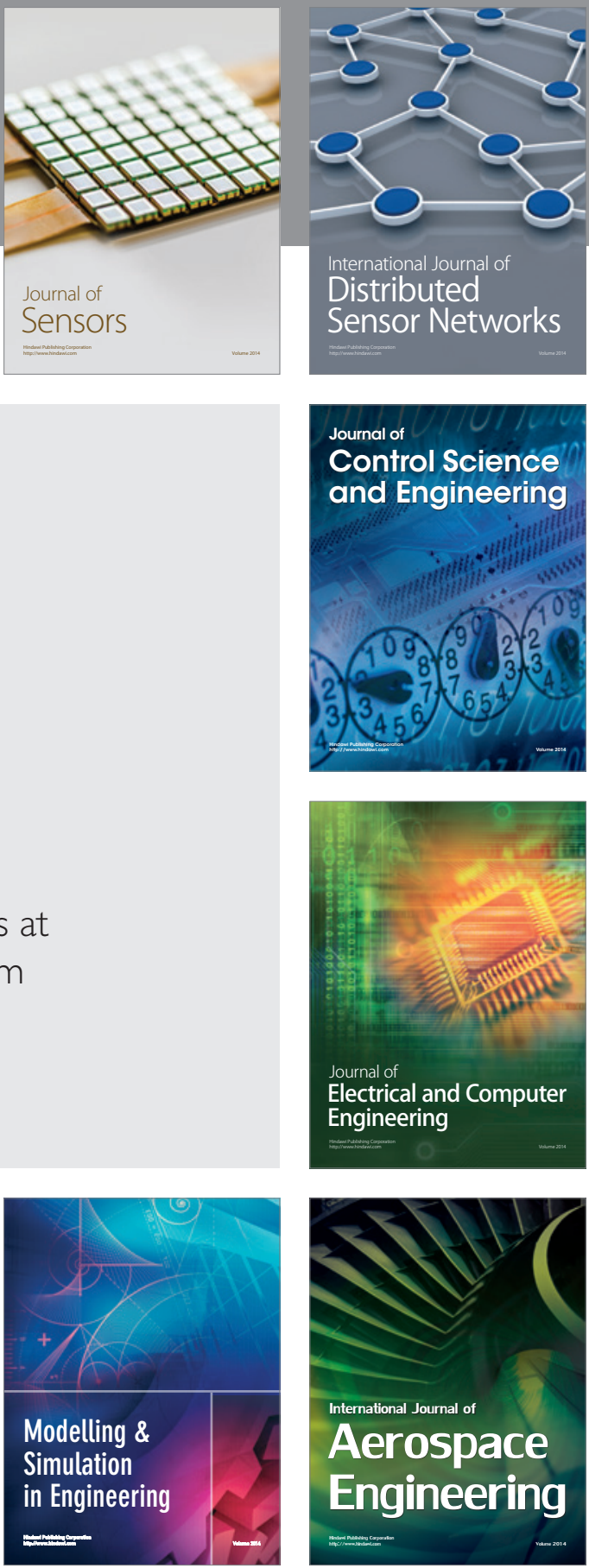

Journal of

Control Science

and Engineering
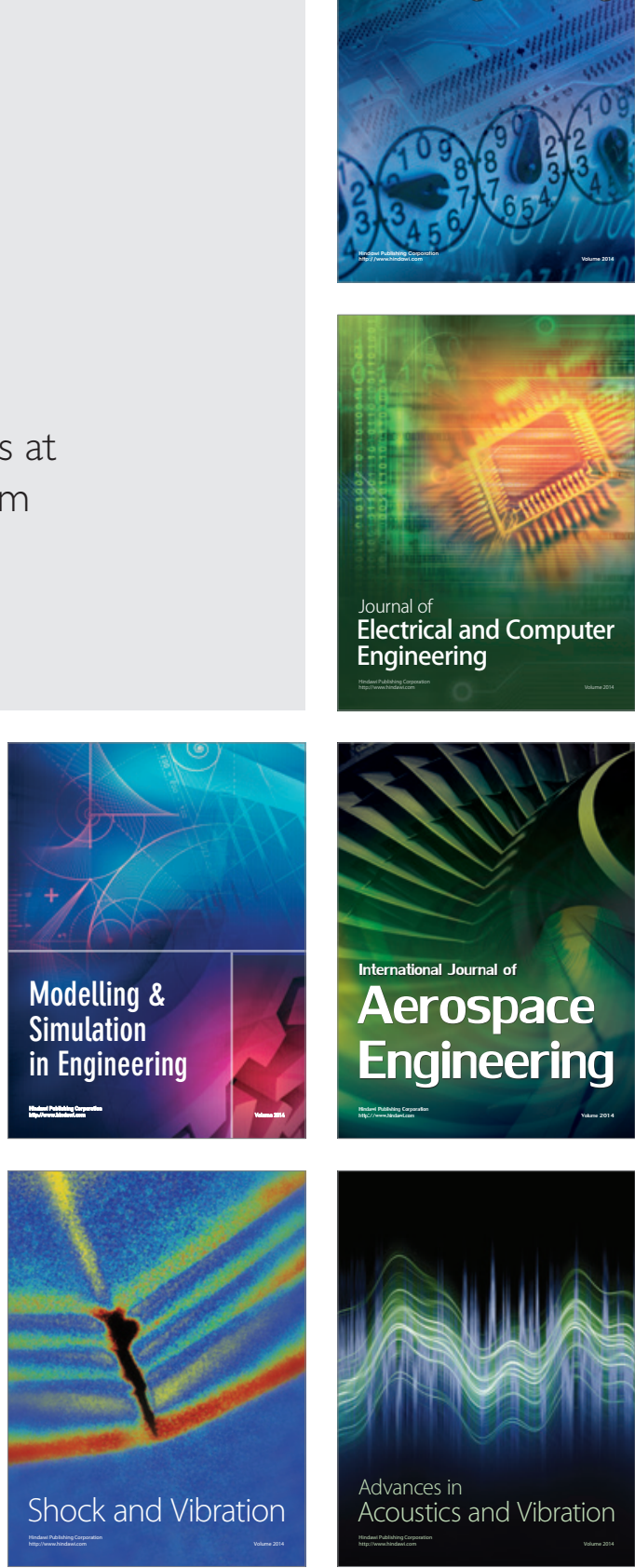